\title{
The Effect of Fiber Orientation on the Toughening of Short Fiber-Reinforced Polymers
}

\author{
David A. Norman, Richard E. Robertson \\ Department of Materials Science and Engineering, University of Michigan, Ann Arbor, Michigan 48109-2136
}

Received 25 November 2002; accepted 13 March 2003

\begin{abstract}
The effect of fiber orientation on the toughening of polymers by short glass fibers generally below their critical length was investigated using specimens with either well-aligned or randomly oriented fibers. The fibers were aligned by an electric field in a photopolymerizable monomer, which was polymerized while the field was still being applied. These materials were fractured with the aligned fibers in three orientations with respect to the crack plane and propagation direction. Specimens with fibers aligned normal to the fracture plane were the most tough, those with randomly oriented fibers were less tough, and those with fibers aligned within the fracture plane were the least tough. The fracture behaviors compared favorably with predictions based on observed processes accounting for fiber orienta-
\end{abstract}

tion. The processes considered were fiber pull-out (including snubbing), fiber breakage, fiber-matrix debonding, and localized matrix-yielding adjacent to fibers bridging the fracture plane. Fibers not quite perpendicular to the fracture plane provided the greatest toughening; these fibers pulled out completely and gave a significant contribution from snubbing. Fibers at higher angles provided less toughening, involving nearly equal contributions from pull-out, breakage, and debonding. Fibers within the fracture plane provided the least toughening, involving debonding alone. (c) 2003 Wiley Periodicals, Inc. J Appl Polym Sci 90: 2740-2751, 2003

Key words: fracture; thermosets; short fibers; orientation; pull-out

\section{INTRODUCTION}

Short fibers are frequently added to polymers to improve elastic modulus, creep resistance, and dimensional stability. ${ }^{1,2}$ Such composites find widespread use because they may be processed with techniques used for unfilled polymers, provided the fiber length is below ca. $10 \mathrm{~mm} .^{2}$ The fracture of polymers is also affected by the addition of short, reinforcing fibers. This behavior is complex, and depends on a number of factors including toughness of the neat polymer, fiber length, and fiber orientation. ${ }^{2-5}$

Flow-induced fiber orientation typically occurs during the processing of reinforced polymers, which leads to anisotropic fracture behavior. Some degree of fiber orientation has been observed in reinforced polymers after injection molding, ${ }^{6-10}$ compression molding, ${ }^{11,12}$ transfer molding ${ }^{13}$ and extrusion, ${ }^{14,15}$ and the ratio of fracture toughnesses of specimens oriented along and transverse to the flow direction has been found to range from 1.4-2.5. ${ }^{6-10,12}$ Although the fracture resistance is enhanced when fibers are perpendicular to the fracture plane, it may approach or even drop below that of the neat polymer when fibers are within the fracture plane. ${ }^{4,5,7}$ Therefore, successful component design with short fiber-reinforced polymers requires

Correspondence to: R. E. Robertson (rer@umich.edu).

Journal of Applied Polymer Science, Vol. 90, 2740-2751 (2003) (C) 2003 Wiley Periodicals, Inc. that the fiber orientation and its effect on fracture behavior be known.

The most insightful studies heretofore have examined well-aligned fibers both perpendicular to or within the fracture plane. ${ }^{10,16,17}$ But few of these have studied fibers at oblique angles in the same systems. The few that have include the work of Lumini and Pavan, who investigated the fracture of nylon with uniformly aligned glass fibers over a range of angles. ${ }^{10}$ But fracture occurred along the direction of fiber orientation at all angles except when the fibers were nearly perpendicular to the fracture plane. Also, Helfet and Harris investigated the fracture of epoxy with either uniformly aligned or randomly oriented metal fibers, ${ }^{18}$ but the use of ductile fibers limits the relevance to systems with brittle fibers, such as glass fibers.

In an attempt to assess the effect of fiber orientation over a range of angles, the fracture of a short glass fiber-reinforced polymer was investigated in which the fibers were either uniformly aligned or randomly oriented. Aligned specimens were prepared by suspending fibers in a nonconductive monomer, aligning the fibers with an electric field, and polymerizing the monomer to capture the aligned microstructure. Nonaligned (random) specimens were prepared by following this procedure without exposure to the electric field. The fracture behavior of these specimens has been compared with calculations based on observed toughening mechanisms, primarily fiber pull-out, fiber breakage, and fiber-matrix debonding. 


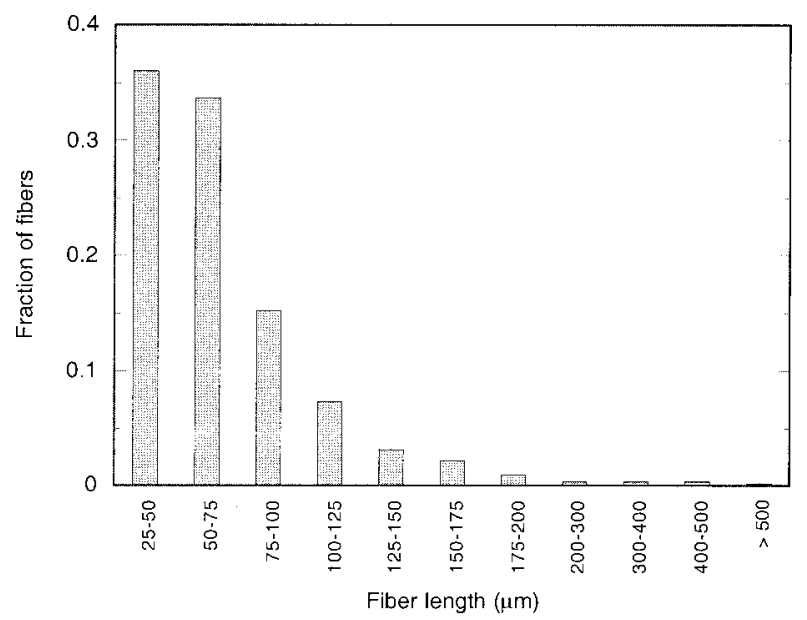

Figure 1 Fiber length distribution of Microglass 3082 milled glass fibers.

\section{EXPERIMENTAL}

\section{Materials}

A mixture of two photopolymerizable monomers were used for the matrix phase; these were urethane dimethacrylate (UDMA) and 1,6-hexanediol dimethacrylate (HDDMA). The monomers were mixed in a $9: 1$ ratio by weight of UDMA to HDDMA to achieve a viscosity of ca. $2.5 \mathrm{~Pa} \cdot \mathrm{s} .{ }^{19}$ Camphorquinone $(0.3 \mathrm{wt} \%)$ and $\mathrm{N}, \mathrm{N}$-dimethylaminoethyl methacrylate $(0.2 \mathrm{wt} \%)$ were added as light-activated initiator and accelerator, respectively. Milled glass fibers (Microglass 3082, Fibertec Inc.) were used as the reinforcement, and these fibers are of the type commonly used to reinforce thermoplastics and thermosets. The fiber length, $l_{f}$, ranged from 25-750 $\mu \mathrm{m}$; the fiber length distribution is shown in Figure 1. The number-average fiber length was $70 \mu \mathrm{m}$, and the weight-average fiber length was $115 \mu \mathrm{m}$. The fiber diameter, $d_{f}$, was $16 \mu \mathrm{m}$. The fibers were used as received, and had not been treated with a size or silane coating. Observation by scanning electron microscopy showed the surface of the fibers to be clean, which should provide a moderately strong bond to the matrix in the absence of moisture.

\section{Specimen preparation}

Aligned specimens were prepared using the following procedure. Glass fibers and monomers were mixed by hand with an agate mortar and pestle. Once mixed, the suspension was heated to ca. $75^{\circ} \mathrm{C}$ and placed under a vacuum to remove entrapped air. After cooling, the suspension was carefully poured into a cell similar to that shown in Figure 2. These cells were constructed from a glass slide $(75 \times 50 \mathrm{~mm})$, polymethylmethacrylate (PMMA) spacers, aluminum electrodes, blocks of a polymeric, open-cell foam, and a PMMA cover. The aluminum electrodes were adhe- sively bonded to the PMMA spacers, and the spacers and foam blocks were bonded to the glass slide, leaving a rectangular cavity. The PMMA spacers were oriented such that the electrodes faced each other on the inside of the cavity. Depending on the desired fiber alignment, the electrodes were positioned on either the sides or ends of the specimen. The spacers determined the height of the cavity, which was enclosed by the PMMA cover. Specimens were exposed to an AC electric field of $0.43 \mathrm{kV} / \mathrm{mm}$, which was generated by a Transco 4B15N3-02 transformer (15 kV at $60 \mathrm{~Hz}$ ) coupled with a Staco 3PN1010 variable autotransformer. After exposure to the field for $1 \mathrm{~min}$, the monomers were photopolymerized using blue light as the specimen remained under the field; this initial cure was performed with a Dentsply Prismetics Lite hand-held light source for approximately $5 \mathrm{~min}$. The foam blocks minimized residual stresses or voids arising from specimen shrinkage during cure. (The foam left an irregular surface finish, which was later ground away.) The initial light cure was followed by light and thermal postcure steps to ensure that all specimens reached a similar state of cure. The specimens were first exposed to a more powerful light source (Dentsply Triad II) for $20 \mathrm{~min}$. The specimens were then heated to $175^{\circ} \mathrm{C}$ under a vacuum for $2 \mathrm{~h}$. This temperature was chosen based on differential scanning calorimetry of the resin system, which showed that the thermal cure was complete ca. $160^{\circ} \mathrm{C}$.

Nonaligned specimens were prepared using the same procedure, except they were not subjected to the electric field prior to polymerization.

In addition to the unfilled resin, specimens were prepared with fiber concentrations of 10, 20, and 30 vol $\%$ for each fiber arrangement. These correspond to 19,34 , and $47 \mathrm{wt} \%$, respectively.

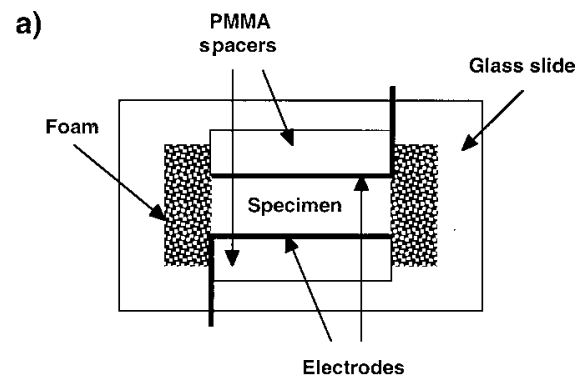

b)

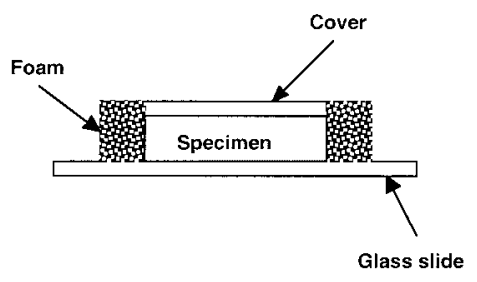

Figure 2 Schematic of a typical cell for preparation of aligned specimens shown in (a) plan view and (b) crosssection. 


\section{Microscopy}

Observations of fracture surfaces and sectioned specimens were made with scanning electron and optical microscopy. Scanning electron microscopy was performed using a Philips XL30FEG microscope. Specimens were first coated with a thin film of carbon to make them electrically conductive. Optical microscopy was performed using an Olympus BH-2 polarizing microscope. Specimens were sectioned with a Buehler Isomet low-speed diamond saw.

To observe fracture and flexural specimens under load, a fixture was fabricated to fit the stage of the optical microscope. Fracture specimens were wedgeloaded, and flexural specimens were loaded in threepoint bending. Specimen geometry was similar to that described for mechanical testing, except the thickness was reduced to 1-2 $\mathrm{mm}$. Both sides of the specimen were polished, and observations were made using transmitted light.

\section{Mechanical testing}

The fracture toughness, flexural modulus, and yield stress were measured. Fracture toughness was obtained from single-edge-notched specimens loaded in three-point bending. Fracture specimens were notched using a thin saw blade, and a precrack was started at the end of the notch with a razor blade. The fracture toughness, $K_{Q}$, was calculated from

$$
K_{Q}=\frac{P_{Q} S}{W H^{3 / 2}} \cdot f(a / H)
$$

where $P_{Q}$ is the load at fracture, $S$ is the span between support pins $(30.3 \mathrm{~mm}), W$ is the specimen width, $H$ is the specimen height, and $a$ is the crack length. The term $f(a / H)$ is given by

$$
f(a / H)=\frac{3(a / H)^{1 / 2}\left[\begin{array}{c}
1.99-(a / H)(1-a / H) \\
\times\left(2.15-3.93 a / H+2.7 a^{2} / H^{2}\right)
\end{array}\right]}{2(1+2 a / H)(1-a / H)^{3 / 2}}
$$

Typical dimensions of the fracture specimens were 35 $\times 6 \times 14 \mathrm{~mm}(\mathrm{~L} \times \mathrm{W} \times \mathrm{H})$, and the ratio $a / H$ was maintained at ca. 0.5 for all specimens, from which $f(a / H)$ had the value of ca. 2.7. Certain specimens were made with slightly smaller dimensions because the depth of the photopolymerization was limited. $K_{Q}$ obtained from eq. (1) is valid for plane-strain fracture toughness, $K_{I c}$, if

$$
2.5\left(\frac{K_{Q}}{\sigma_{Y}}\right)^{2} \leq a, W, \text { and }(H-a)
$$

where $\sigma_{Y}$ is the yield stress. Dimensions for all fracture specimens were such that eq. (3) was satisfied. A more detailed examination of the validity of fracture mechanics for the current system will be addressed later.

The flexural modulus was obtained from unnotched specimens also in three-point bending. The flexural modulus, E, was calculated using

$$
E=\frac{S^{3}}{4 W H^{3}} \frac{\Delta P}{\Delta D}
$$

where $\Delta P / \Delta D$ is the slope of the load vs. displacement curve within the linear region. The dimensions of the flexural specimens were $35 \times 14 \times 3 \mathrm{~mm}(\mathrm{~L} \times \mathrm{W} \times \mathrm{H})$, and $S$ was $30.3 \mathrm{~mm}$.

The yield stress was obtained from the compression of cylindrical specimens between parallel plates. Specimen length and diameter were 12 and $8.2 \mathrm{~mm}$, respectively. Molybdenum disulfide grease was used as a lubricant between the specimen and the plates. Yield stress was measured in compression because specimens failed in flexure before obvious yielding occurred.

All measurements were made with a 4502 Instron at a crosshead speed of $0.5 \mathrm{~mm} / \mathrm{min}$. Results have been presented as the average of six equivalent specimens. Specimen dimensions in the above equations were the average of four measurements.

\section{RESULTS}

\section{Microstructures and fiber angle distribution}

Typical microstructures visible on fracture surfaces with 20 vol \% glass fibers are shown in Figure 3. For the nonaligned specimens [Fig. 3(a)], no preferred fiber orientation was observed. For the aligned specimens [Fig. 3(b)], fibers were oriented in the direction of the applied field and frequently formed structures in which the aligned fibers were arranged end to end. Such structures were typically formed from fibers less than ca. $100 \mu \mathrm{m}$ in length. Fiber alignment was uniform throughout the specimen.

The fiber angle distribution was measured from optical micrographs of polished sections with $10 \mathrm{vol} \%$ glass fibers. The analysis was performed using the public domain NIH Image program ${ }^{20}$ on digitized images. Planar fiber angle distributions (i.e., measured directly from the surface of polished sections) for the nonaligned and aligned specimens are shown in Figure 4 . Without alignment, the fraction of fibers within each $5^{\circ}$ range was nearly the same and approached the value estimated for randomly oriented fibers, 5.6\%. With alignment, $66 \%$ of the fibers were within $5^{\circ}$ of the applied field direction, and $98 \%$ were within $20^{\circ}$. The planar fiber angle distributions were converted to three dimensions by assuming the distributions to be 
a)

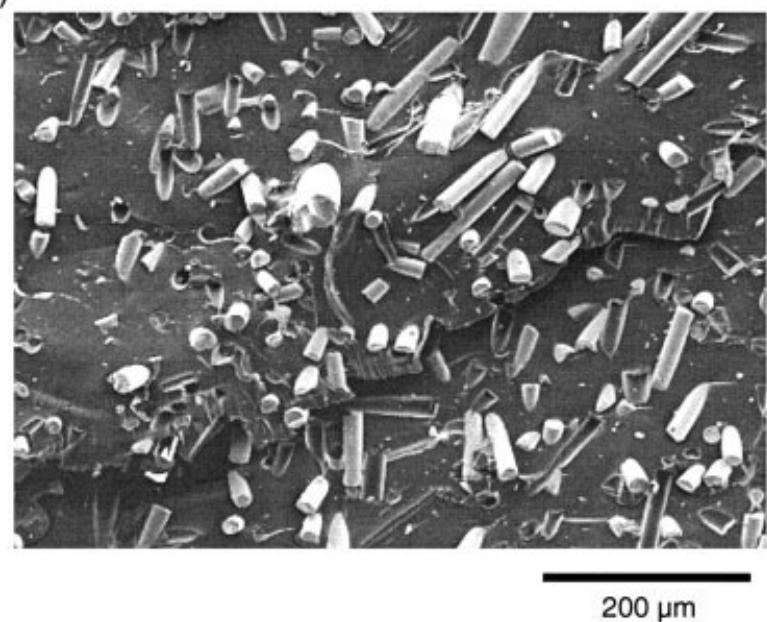

b)

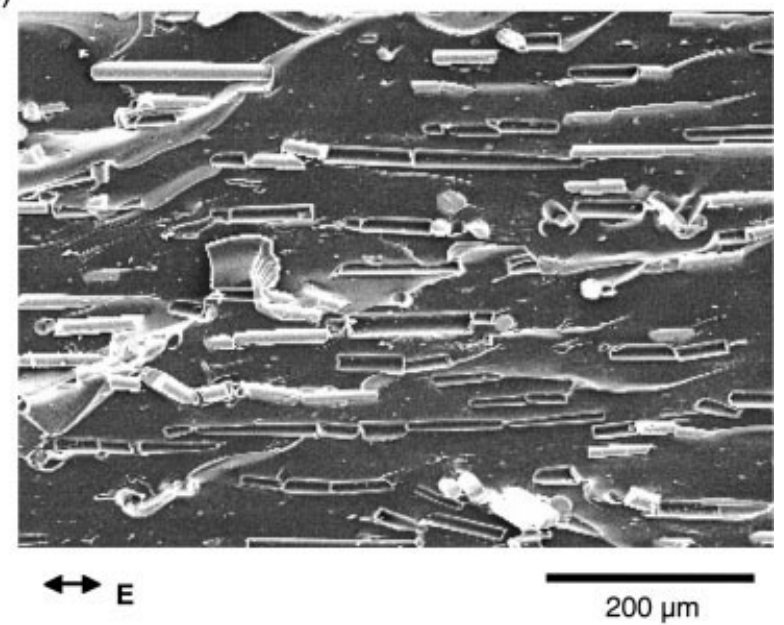

Figure 3 Fracture surfaces of (a) random and (b) aligned specimens showing typical fiber arrangements. Fiber concentration is $20 \mathrm{vol} \%$ for both micrographs.

symmetric about the field axis, giving ellipsoidal symmetry with alignment and spherical symmetry without alignment. The conversion of the planar distributions to three dimensions decreased the fraction of fibers closely aligned with the field axis and slightly increased the fraction of fibers at high angles to the field axis from those in Figure 4.

\section{Fracture toughness and fracture energy}

For the fracture experiments, three different fiber orientations were investigated (Fig. 5), in addition to nonaligned specimens. These were given names that describe the relationship between the direction of alignment and the crack plane and growth. With the fibers perpendicular to the fracture plane [Fig. 5(a)], the specimen was termed "normal-aligned." The other two alignments have fibers within the fracture plane,

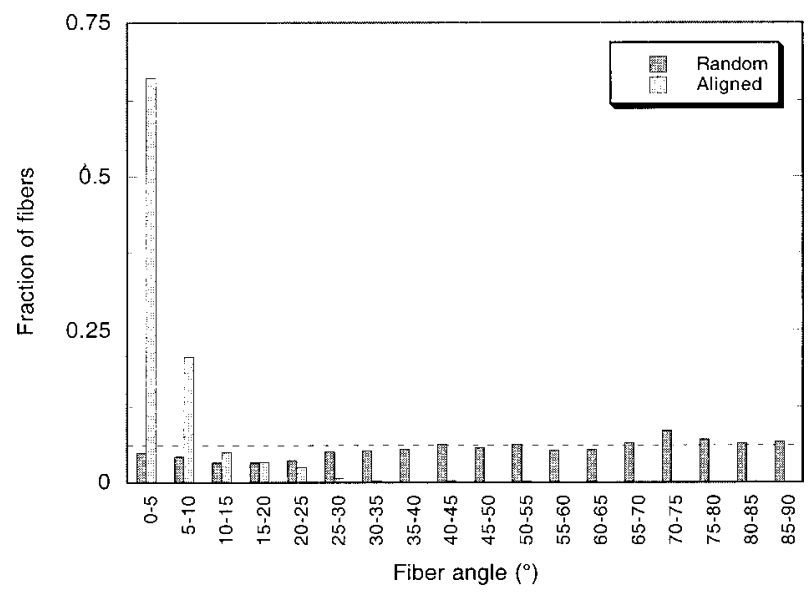

Figure 4 Planar fiber angle distribution for random and aligned specimens with $10 \mathrm{vol} \%$ fibers. The direction of alignment corresponds to $0^{\circ}$. The dashed line is the prediction for a random fiber angle distribution. and to distinguish between the two, the orientation of fibers within the fracture plane was related to the direction of crack propagation. Thus, the two are termed "in-plane parallel-aligned" [Fig. 5(b)] and "inplane normal-aligned" [Fig. 5(c)].

The fracture toughness, $K_{I c}$ and its dependence on fiber concentration and arrangement is shown in Figure 6 . Although the only fiber concentrations studied were $0,10,20$, and $30 \mathrm{vol} \%$, the data in Figure 6 have been shifted about these concentrations to allow the fracture toughness and error bars for each fiber orientation to be distinguished. The fracture toughness of the neat resin was $1.1 \mathrm{MPa} \cdot \mathrm{m}^{1 / 2}$. With nonaligned fibers, the average fracture toughness increased by a factor of ca. 2.2 from 0 to $30 \mathrm{vol} \%$; the largest increase occurred between 10 and $20 \mathrm{vol} \%$. At 10 vol \% glass fibers, all fiber orientations had about the same fracture toughness. At 20 and $30 \mathrm{vol} \%$, the normalaligned specimens were tougher than the nonaligned, and both in-plane alignments were less tough than the nonaligned. The two in-plane alignments displayed the same fracture toughness over all fiber concentrations. The greatest difference in fracture toughness between the orientations, a factor of two, was ob-

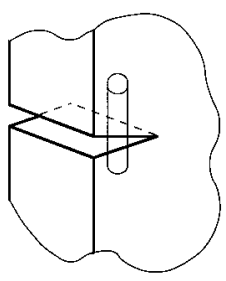

a) Normal

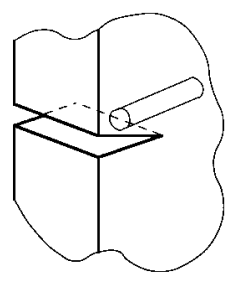

b) In-plane parallel

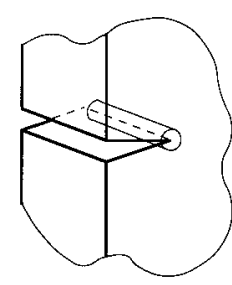

c) In-plane normal
Figure 5 Schematic of the three fiber alignments investigated with respect to the crack geometry: (a) normalaligned, (b) in-plane parallel-aligned, and (c) in-plane normal-aligned. 


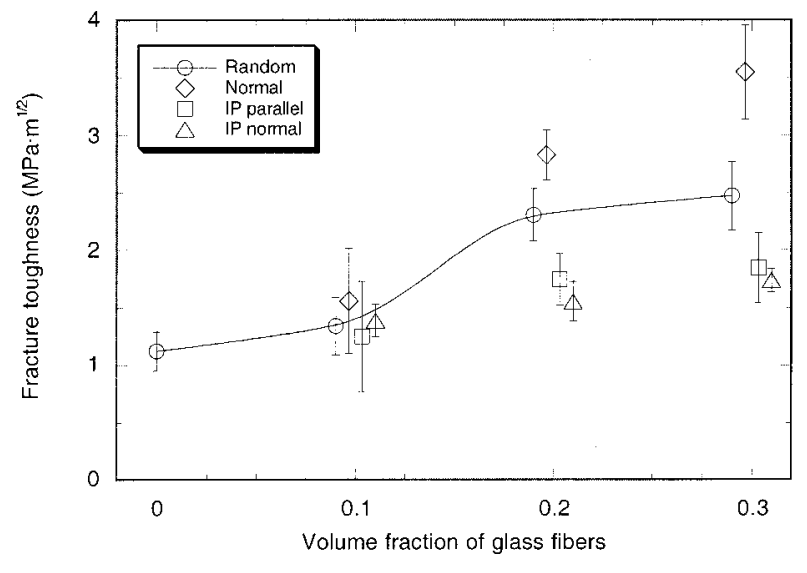

Figure 6 The effect of fiber concentration and orientation on fracture toughness. Data at a common fiber concentrations have been shifted horizontally to distinguish between fiber orientations. The line connecting the random data is included only as a guide.

served at $30 \mathrm{vol} \%$ glass fibers. The average fracture toughness was $1.7-1.8 \mathrm{MPa} \cdot \mathrm{m}^{1 / 2}$ for the two in-plane alignments, $2.5 \mathrm{MPa} \cdot \mathrm{m}^{1 / 2}$ for nonaligned specimens, and $3.5 \mathrm{MPa} \cdot \mathrm{m}^{1 / 2}$ for the normal-aligned specimens.

Fracture toughness results were also converted to fracture energy, $G_{I c}$. For the nonaligned specimens, fracture energy was calculated from ${ }^{21}$

$$
G_{I c}=\frac{K_{I c}^{2}}{E}\left(1-\nu^{2}\right)
$$

where $E$ is the elastic modulus and $\nu$ is Poisson's ratio. The modulus was measured in flexure, and the results are given in Figure 7. Poisson's ratio for specimens with nonaligned fibers was estimated using the rule of mixtures,

$$
\nu_{c}=\nu_{f} V_{f}+\nu_{m}\left(1-V_{f}\right)
$$

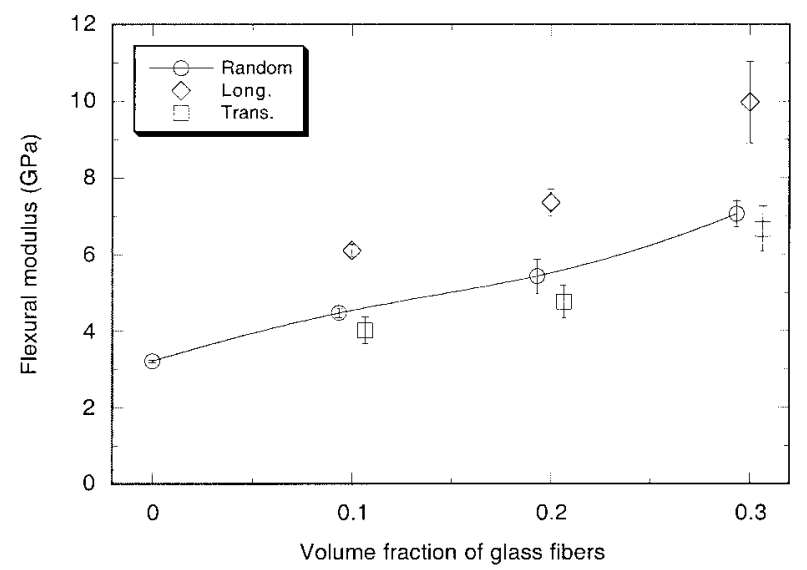

Figure 7 The effect of fiber concentration and orientation on flexural modulus. Data at a common fiber concentrations have been shifted horizontally to distinguish between fiber orientations. The line connecting the random data is included only as a guide.

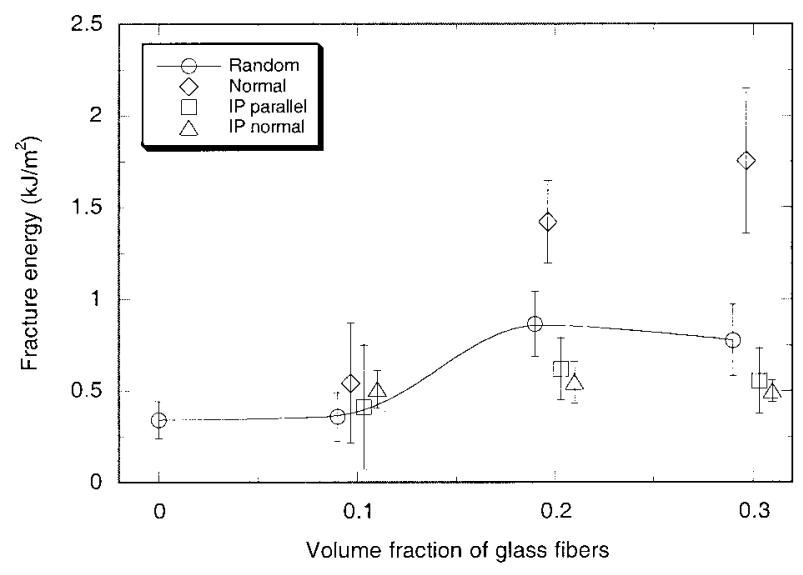

Figure 8 The effect of fiber concentration and orientation on fracture energy. Data at a common fiber concentrations have been shifted horizontally to distinguish between fiber orientations. The line connecting the random data is included only as a guide.

where $V_{f}$ is the fiber volume fraction, and the subscripts $c, m$, and $f$ refer to composite, matrix, and fibers. Values of 0.22 and 0.38 were used for Poisson's ratio of the fibers and matrix, respectively. For the aligned specimens, fracture energy was calculated using orthotropic fracture mechanics ${ }^{22}$ because alignment caused the elastic behavior to become anisotropic. The flexural modulus was measured for specimens aligned along and transverse to the long axis of the beam, and these orientations were designated longitudinal and transverse. The longitudinal modulus was ca. 1.5 times greater than the transverse modulus at all fiber concentrations (Fig. 7). Poissons ratios for the aligned specimens were estimated using rules of mixture for a unidirectional fiber composite. ${ }^{23}$

The fracture energy and its dependence on fiber concentration and arrangement is shown in Figure 8. The fracture energy of the neat resin was $340 \mathrm{~J} / \mathrm{m}^{2}$. For specimens with nonaligned fibers, the average fracture energy increased by a factor of ca. 2.5 from 0 to 20 vol \%, and the fracture energy remained about the same between 20 and $30 \mathrm{vol} \%$. Differences in fracture energy resulting from fiber alignment were similar to trends observed for fracture toughness. At $10 \mathrm{vol} \%$ glass fibers, all fiber orientations displayed about the same fracture energy. At 20 and $30 \mathrm{vol} \%$, the normalaligned specimens exhibited higher fracture energy than the nonaligned, and both in-plane alignments displayed even lower fracture energy. The two inplane alignments displayed about the same fracture energy at all fiber concentrations. The largest difference in fracture energy between the orientations, nearly a factor of four, was observed at $30 \mathrm{vol} \%$. The average fracture energy was $500-550 \mathrm{~J} / \mathrm{m}^{2}$ for the two in-plane alignments, $780 \mathrm{~J} / \mathrm{m}^{2}$ for the nonaligned specimens, and $1910 \mathrm{~J} / \mathrm{m}^{2}$ for the normal-aligned specimens. 


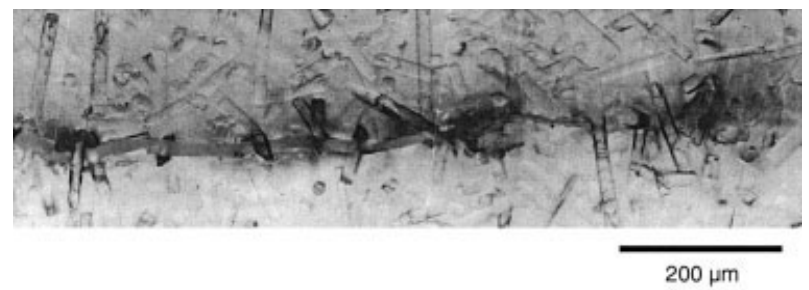

Figure 9 Typical damage within the process zone of a nonaligned specimen. Fiber concentration is $20 \mathrm{vol} \%$.

\section{Observations of damage mechanisms within the process zone}

A number of damage mechanisms were observed in the process zone of short fiber-reinforced polymers; the primary mechanisms were fiber pull-out, fiber breakage, and fiber-matrix debonding. Typical damage mechanisms in the vicinity of a wedge-loaded crack tip can be seen in Figure 9 for a nonaligned specimen with $20 \mathrm{vol} \%$ fibers. The crack tip is located at the far right of Figure 9, and the portion of the crack shown extends ca. $1 \mathrm{~mm}$ behind the crack tip. Transmitted light was used to observe fibers below the surface of the specimen. Fibers bridge the crack at several locations in Figure 9 and either pull-out from the matrix or break. Fiber-matrix debonding is also seen as a dark outline around fibers.

Fibers that bridged the crack either pulled-out or broke, depending on the fiber orientation and the embedment length. Complete pull-out occurred for nearly all fibers that were less than ca. $10^{\circ}$ from being perpendicular to the fracture plane. For fibers at larger angles, pull-out was restricted to those that were embedded less than about one fiber diameter into the matrix; fiber breakage occurred for fibers that were embedded more deeply.

For fibers that were oriented nearly within the fracture plane, fiber breakage was less apparent, but instead, fiber-matrix debonding appeared to dominate. Although less obvious away from the crack tip, most of the fibers within a region extending ca. $100 \mu \mathrm{m}$ above and below the crack plane are debonded. The debonding occurred regardless of fiber orientation or whether or not the fibers intersected the crack plane.

Although difficult to see in Figure 9, localized matrix yielding occurred on the fracture surface adjacent to fibers that bridged the crack at oblique angles. Localized matrix yielding was apparent in scanning electron micrographs of fracture surfaces, where gaps between the matrix and fibers were frequently observed around broken fibers.

\section{Compressive yield stress}

The compressive yield stress of specimens with nonaligned fibers is given in Figure 10. The yield stress of the neat resin was $130 \mathrm{MPa}$. The yield stress increased with fiber concentration, reaching $165 \mathrm{MPa}$ at 30 vol \%.

\section{DISCUSSION}

The resin was toughened by the addition of short glass fibers. For specimens with nonaligned fibers, the fracture toughness increased monotonically to $30 \mathrm{vol} \%$ (Fig. 6); the fracture energy also increased with fiber concentration but appeared to level off above $20 \mathrm{vol} \%$ (Fig. 8). This behavior is similar to that of previous studies on low toughness polymers reinforced with short glass fibers. ${ }^{7,17,24}$ At 10 vol \%, all fiber arrangements exhibited about the same toughness, but at higher concentrations, the fracture behavior depended on fiber orientation. At 20 and $30 \mathrm{vol} \%$, the normalaligned specimens were tougher than the nonaligned specimens, and both in-plane alignments were less tough. The anisotropy of the fracture behavior was similar to previous studies on uniformly aligned short fiber-reinforced polymers. ${ }^{10,16,17}$

Three main damage mechanisms were observed in the process zone: fiber pull-out, fiber breakage, and fiber-matrix debonding. Fiber pull-out or fiber breakage occurred for fibers that bridged the crack. Complete fiber pull-out occurred for fibers that were nearly perpendicular to the fracture plane or had at least one end embedded less than about one fiber diameter into the matrix. Fiber breakage occurred for fibers that bridged the crack at oblique angles and had both ends embedded more than about one fiber diameter into the matrix. Localized matrix yielding adjacent to fibers on the fracture surface often accompanied fiber pullout and breakage. Fiber-matrix debonding occurred for nearly all fibers sufficiently close to the crack plane, and was the only available toughening mechanism for fibers oriented within the fracture plane. These mechanisms have also been noted by others. ${ }^{7,25-27}$

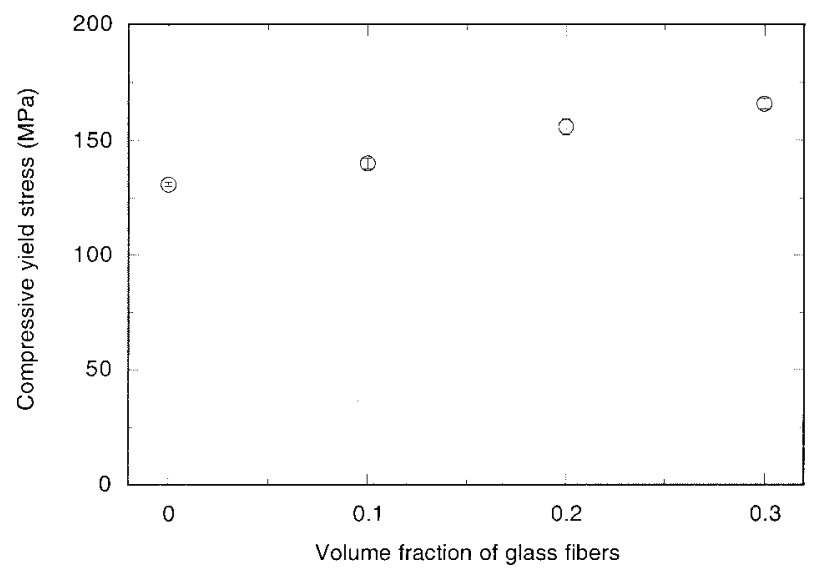

Figure 10 The effect of fiber concentration on compressive yield stress of nonaligned composites. 


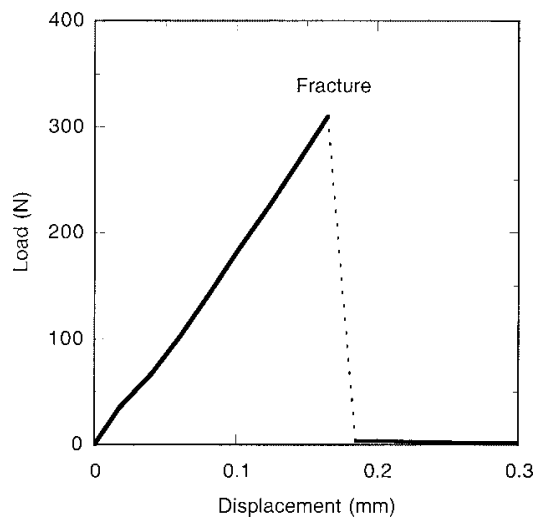

Figure 11 Typical load vs. displacement curve for a fracture experiment. The curve shown is for a specimen with 20 vol $\%$ of randomly oriented fibers.

The fracture behavior of random and aligned short fiber composites may be compared with predictions based on processes observed during fracture.

\section{Validity of linear elastic fracture mechanics}

The applicability of linear elastic fracture mechanics (LEFM) to short fiber composites has been investigated a number of times. ${ }^{6,8,9,28-30}$ Short fibers tend to increase the size of the process zone around the crack tip, which must remain smaller than the specimen dimensions to obtain valid plane-strain fracture toughnesses.

The validity of LEFM in the current system is suggested by the following. First, elastic behavior is indicated by the essentially linear load versus displacement curve up to fracture (Fig. 11). Second, the process zone size is estimated to be less than the smallest specimen dimension. Equation (3), which is intended for homogeneous materials, predicts the maximum process zone size to be ca. $0.6 \mathrm{~mm}$. An expression for randomly oriented short fiber composites, $W$, which is more appropriate for the current system, is ${ }^{30}$

$$
W=\frac{9 \pi}{32} \frac{E}{\left(1-\nu^{2}\right) \sigma_{u}} \frac{\bar{l}_{f}}{4}
$$

where $\sigma_{u}$ is the ultimate tensile strength, and $\bar{l}_{f}$ is the average fiber length. Using the failure stress in flexure (ca. $100 \mathrm{MPa}$ ) for $\sigma_{u}$ and the weight average fiber length $(115 \mu \mathrm{m})$ for $\bar{l}_{f}$, the maximum process zone size is predicted to be ca. $2.2 \mathrm{~mm}$. Both estimates of zone size are less than the smallest dimension for all specimens (typically $6 \mathrm{~mm}$ ). Third, fracture toughness was constant over specimen heights ranging from 6-18 $\mathrm{mm}$ (Fig. 12), indicating again that the process zone is smaller than $6 \mathrm{~mm}$. The experiments for Figure 12 were performed on nonaligned specimens with a fiber concentration of $20 \mathrm{vol} \%$, with the ratio $a / H$ maintained at ca. 0.5.

\section{Estimation of critical fiber length}

The critical fiber length is twice the length over which the transfer of load from the matrix is sufficient for the peak tensile stress in the fiber to reach the ultimate tensile strength. Fiber pull-out lengths in excess of 150 $\mu \mathrm{m}$ were observed by optical microscopy of the fracture surfaces; this indicates the critical fiber length to be at least as great as $300 \mu \mathrm{m}$, assuming pull-out of the shorter portion of the fiber bridging the fracture plane. Fewer than ca. $0.5 \%$ of fibers were above this length. The critical fiber length was also estimated using a shear lag model, assuming a constant shear stress across the fiber-matrix interface, $\tau_{i \cdot}{ }^{23}$ This gives

$$
l_{f c}=\frac{\sigma_{f u} d_{f}}{2 \tau_{i}}
$$

where $l_{f c}$ is the critical fiber length, $d_{f}$ is the fiber diameter, and $\sigma_{f u}$ is the ultimate tensile strength of the fiber. A typical value of $\tau_{i}$ for a glass fiber reinforced polymer is ca. $20 \mathrm{MPa}^{23}$ This gives a critical fiber length of $800 \mu \mathrm{m}$, and all fibers were below this length. The use of the shear yield stress of the resin (ca. 65 MPa) as an upper limit for $\tau_{i}$ still gives a critical fiber length of $250 \mu \mathrm{m}$, and fewer than $1 \%$ of the fibers were above this length. Therefore, almost all, if not all, of the fibers can be assumed to be below their critical length.

\section{Estimation of energy absorbed during fracture}

The fracture energy of random and aligned short fiber composites can be estimated by summing the contributions from the observed mechanisms, taking fiber orientation into account. The processes considered were fiber pull-out, fiber breakage, fiber-matrix debonding, and localized matrix yielding adjacent to

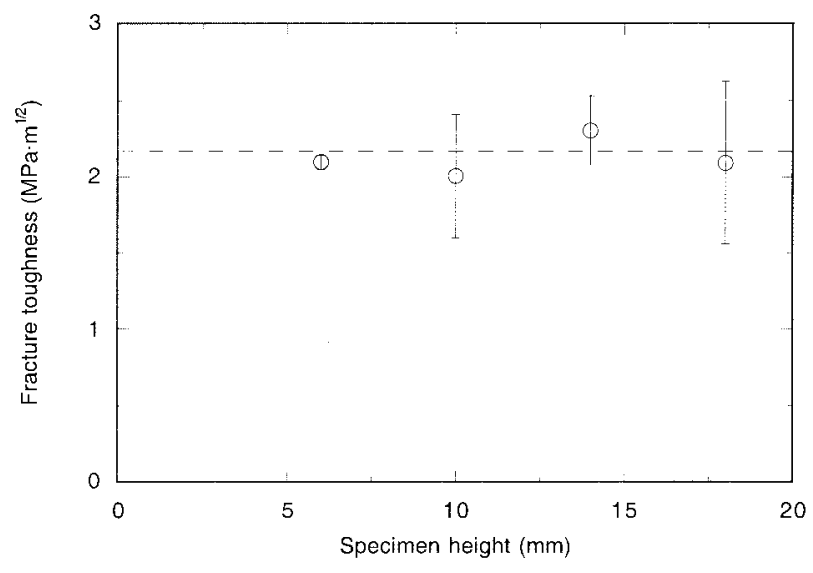

Figure 12 The effect of specimen height on fracture toughness. Fiber concentration is $20 \mathrm{vol} \%$, and fibers are randomly oriented. Specimen width and the ratio of crack length to specimen height $(a / H)$ are the same for all specimens. 
fibers on the fracture plane. A brief overview of concepts used throughout the calculations is presented before describing how the different mechanisms were addressed.

The contributions from all mechanisms except fiber-matrix debonding depend on the embedded fiber length, the number of fibers that bridge the crack, and localized deformation around fibers bridging the crack at oblique angles. The embedded fiber length, $l_{e}$, refers to the length of the shorter segment of the fiber intersected by the crack plane. The distribution of embedded fiber lengths has been calculated from the fiber length distribution (Fig. 1) assuming that the fracture plane has an equal probability of intersecting a fiber at any location along its length. The average embedded fiber length is significantly less than the fiber length, and is estimated to be $l_{f} / 4$. Roughly $70 \%$ of the fibers have embedded lengths less than $20 \mu \mathrm{m}$, and about $90 \%$ of the fibers have embedded lengths less than $35 \mu \mathrm{m}$.

The number of fibers intersected by the fracture plane can be estimated as a function of $\theta$, the polar angle between the fiber axis, and the normal to the fracture plane. Consider a cube of unit dimensions containing $N_{V}$ fibers of average length $\bar{l}_{f}$ with their centers randomly distributed in space. Assume periodic boundary conditions, in which fibers that protrude from a surface reenter on the opposite surface. The number of fibers in the unit cube is

$$
N_{V}=\frac{V_{f}}{\pi r_{f}^{2} \bar{l}_{f}}
$$

where $V_{f}$ is the volume fraction of fibers and $r_{f}$ is the fiber radius. If the fracture plane is parallel to the cube base, the probability of a fiber of length $\bar{l}_{f}$ being intersected by the plane is approximately

$$
p(\theta)=\bar{l}_{f} \cos \theta
$$

Although eq. (10) underestimates the probability of fibers being intersected by the fracture plane as $\theta$ approaches $90^{\circ}$, this will have a negligible effect on estimating the fracture energy. For a fiber of average length, eq. (10) only underestimates this probability for fibers oriented within ca. $8^{\circ}$ of the fracture plane. Fibers in this orientation toughen primarily by fibermatrix debonding, which was observed to occur for nearly all fibers within ca. $100 \mu \mathrm{m}$ of the fracture plane. Hence, the contribution from debonding does not rely on fibers being intersected by the fracture plane.

The density of fibers intersected by the fracture plane per unit area as a function of $\theta$ is then

$$
\rho(\theta)=f(\theta) N_{V} p(\theta)=\frac{V_{f}}{\pi r_{f}^{2}} f(\theta) \cos \theta
$$

where $f(\theta)$ describes the polar fiber angle distribution within the cube. Therefore, the number of fibers within an angular region $d \theta$ about $\theta$ is $\rho(\theta) d \theta$. Because the fiber length cancels when eqs. (9) and (10) are multiplied, $\rho(\theta)$ is independent of fiber length.

Several phenomena relating to toughening occur when fibers bridge the crack at oblique angles. First, fibers that pull out from the matrix at oblique angles are bent into an S-shape as the crack opens, which gives rise to a bending moment along the fiber. This may cause the fiber to break if its flexural strength is exceeded. In the current system, the occurrence of fiber breakage is predicted to arise only from bending because the fibers are generally below their critical length. Second, fiber bending during pull-out gives rise to increased friction as the fiber exits the matrix, known as snubbing. ${ }^{31}$ However, snubbing in systems with brittle fibers is limited because fibers bend very little before breaking. Third, bending of the fibers is accompanied by localized matrix deformation that may result in matrix yielding.

These phenomena were included in the model developed by Morton and Groves for fibers bridging a crack at oblique angles. ${ }^{32}$ This model allows the bending moment along the fiber and the extent of matrix yielding to be calculated as a function of fiber angle and crack separation. However, the original model has been slightly modified. Morton and Groves ${ }^{32}$ used the Vicker's hardness of the matrix as a measure of matrix plasticity, and the current analysis uses the compressive yield stress of nonaligned composites instead (Fig. 10). This is intended to account for the constraint of matrix plasticity from nearby fibers with varying fiber concentration. The compressive yield stress is slightly less than the Vicker's hardness of the matrix (190 MPa) over the range of fiber concentration observed.

Whether fibers pull out completely or break has been estimated from the Morton and Grove's model ${ }^{32}$ by comparing the bending moment along the fiber with the distance over which matrix yielding occurs, $q$. The model predicts that the bending moment increases monotonically, and that the distance over which the matrix yielding occurs reaches a maximum with increasing crack separation, $h$. If the bending moment exceeds the flexural strength of the fiber (ca. $2 \mathrm{GPa}$ ) before $q$ reaches a maximum, the fiber is assumed to break; otherwise the fiber is assumed to pull out completely. The switch between fiber pull-out and breakage occurs at the critical fiber angle, $\theta_{c}$, which is $7-8^{\circ}$ over the range of fiber concentration investigated. Fiber breakage occurs at the critical crack separation, $h_{c}$, which is typically between 3-10 $\mu \mathrm{m}$. Fibers are predicted to break at a depth roughly equivalent to a fiber diameter. The maximum depth at which fibers break is estimated to be ca. $20 \mu \mathrm{m}$ at an angle of ca. $20^{\circ}$, and approaches zero at $90^{\circ}$. The predictions of the 
angle and embedded length at which fibers break agree with observations of the process zone (Fig. 9).

Fiber pull-out

The fiber pull-out energy is the frictional work spent to extract the fiber from the matrix. Fibers with $\theta \leq \theta_{c}$ pull out completely. Fibers with $\theta>\theta_{c}$ pull-out if their embedded length, $l_{e}$, is less than $q($ ca. $20 \mu \mathrm{m})$; those embedded more deeply break at a depth of $q$, and this portion of the fiber pulls out. The frictional force between the fiber and matrix may arise from one of two sources. For $l_{e}>q$, the friction arises from shrinkage stresses generated from curing the matrix. For $l_{e} \leq q$, the friction arises from snubbing, assuming that the stress on the fiber equals the composite yield stress over the length $q$. ( $q$ can be estimated from Morton and Groves's model. ${ }^{32}$ ) This stress acts only on the side of the fiber that is pulled against the matrix. For a single fiber at an angle $\theta \leq \theta_{c}$ and $l_{e}>q$, the pull-out work (including snubbing) is

$$
\begin{aligned}
W_{P O, a}=\mu \pi r_{f}\left\{\sigma_{Y} \int_{0}^{q(\theta)} l d l+\sigma_{Y} q(\theta) \int_{q(\theta)}^{l_{e}>q(\theta)} d l\right. \\
\left.+2 \sigma_{r} \int_{q(\theta)}^{l_{e}>q(\theta)}[l-q(\theta)] d l\right\} \\
=\mu \pi r_{f}\left\{\sigma_{Y} l_{e} q(\theta)+\sigma_{r}\left[l_{e}-q(\theta)\right]^{2}\right\}
\end{aligned}
$$

where $\mu$ is the coefficient of friction between the fiber and matrix, $\sigma_{Y}$ is the composite yield stress, $l$ is the length along the embedded fiber, and $\sigma_{r}$ is the radial compressive stress on the fiber generated during cure. For a single fiber that breaks $\left(\theta>\theta_{c}\right.$ and $\left.l_{e}>q\right)$, the pull-out work is

$$
W_{P O, b}=\mu \pi r \sigma_{Y} \int_{0}^{q(\theta)} l d l=\frac{\mu \pi r_{f} \sigma_{Y} q^{2}(\theta)}{2}
$$

because the fibers break and pull-out over an embedded length of $q$. For a single fiber with $l_{e} \leq q$, the pull-out work is

$$
W_{P O, c}=\mu \pi r_{f} \sigma_{Y} \int_{0}^{l_{l} \leq q(\theta)} l d l=\frac{\mu \pi r_{f} \sigma_{Y} l_{e}^{2}}{2}
$$

The pull-out contribution from all fibers has been calculated with the above equations. To account for all fiber orientations and lengths, eq. (11) and the distribution of embedded fiber lengths, $g\left(l_{e}\right)$, have been included. The total contribution from fiber pull-out for $\theta \leq \theta_{c}$ using eqs. (11), (12), and (14) is

$$
\begin{aligned}
G_{P O, a}= & \frac{\mu V_{f}}{r_{f}} \int_{0^{\circ}}^{\theta_{c}} f(\theta) \cos \theta\left(\frac{\sigma_{Y}}{2} \int_{0}^{q(\theta)} g\left(l_{e}\right) l_{e}^{2} d l_{e}\right. \\
& \left.+\int_{q(\theta)}^{\infty} g\left(l_{e}\right)\left\{\sigma_{Y} l_{e} q(\theta)+\sigma_{r}\left[l_{e}-q(\theta)\right]^{2}\right\} d l_{e}\right) d \theta
\end{aligned}
$$

and the contribution from pull-out for $\theta>\theta_{c}$ using $3.11,3.13$, and 3.14 is

$$
\begin{array}{r}
G_{P O, b}=\frac{\mu \sigma_{Y} V_{f}}{2 r_{f}} \int_{\theta_{c}}^{90^{\circ}} f(\theta) \cos \theta\left[\int_{0}^{q(\theta)} g\left(l_{e}\right) l_{e}^{2} d l_{e}\right. \\
\left.+q^{2}(\theta) \int_{q(\theta)}^{\infty} g\left(l_{e}\right) d l_{e}\right] d \theta
\end{array}
$$

The compressive stress exerted by the matrix on the fibers was estimated to be ca. $27 \mathrm{MPa}$ using the model developed by Harris. ${ }^{33}$ Equation (15) assumes that the only pull-out contribution from broken fibers is that from the broken ends that are embedded a depth $q$.

Fiber breakage

The energy contribution from fiber breakage is estimated to be the elastic energy stored in the fiber when it breaks. Fiber breakage occurs for $\theta>\theta_{c}$ and $l_{e}>q$. The elastic energy in the fiber at failure, $U_{f u}$, is estimated from the area under the stress-strain curve of the fiber times the average fiber volume $\pi r_{f}^{2} \bar{l}_{f}$, where $\bar{l}_{f}$ is the average fiber length. The fiber breakage contribution from all fibers has been calculated by including eq. (11) and the distribution of embedded fiber lengths, which gives

$$
G_{B}=U_{f u} \bar{l}_{f} V_{f} \int_{\theta_{c}}^{90^{\circ}} f(\theta) \cos \theta\left[\int_{q(\theta)}^{\infty} g\left(l_{e}\right) d l_{e}\right] d \theta
$$

The elastic energy stored in the fibers has been estimated to be ca. $30 \mathrm{MJ} / \mathrm{m}^{3}$, assuming the fibers break at a stress of $2 \mathrm{GPa}$.

\section{Matrix yielding}

The energy from matrix yielding is estimated to be the work of plastic strain times the volume over which yielding occurs. The work of plastic strain, $W_{p}$, has been estimated from the area under the stress-strain curve of the neat resin. The yielded volume is assumed to be a right triangle adjacent to each fiber with hypotenuse $q$ and the fracture plane as one side with a thickness of one fiber diameter. For fibers with $l_{e}>q$, this gives 


$$
V_{Y}\left(\theta, l_{e}\right)=d_{f} q^{2}(\theta) \cos \theta \sin \theta
$$

and for $l_{e} \leq q, V_{Y}$ is

$$
V_{Y}\left(\theta, l_{e}\right)=d_{f} l_{e}^{2} \cos \theta \sin \theta
$$

This yielded volume is generated on each side of the fracture plane where the fiber is embedded in the matrix.

Taking the fiber orientation and embedded length distributions into account, the contribution from matrix yielding is estimated to be

$$
\begin{aligned}
G_{Y}= & \frac{2 W_{p} V_{f}}{\pi r_{f}} \int_{0^{\circ}}^{90^{\circ}} f(\theta) \cos ^{2} \theta \\
& \times \sin \theta\left[\int_{0}^{q(\theta)} g\left(l_{e}\right) l_{e}^{2} d l_{e}+q^{2}(\theta) \int_{q(\theta)}^{\infty} g\left(l_{e}\right) d l_{e}\right] d \theta
\end{aligned}
$$

Because the matrix is strongly plowed by the fiber, the plastic strain within the yielded volume will be on the order of $50 \%$, where $W_{p}$ is ca. $65 \mathrm{MJ} / \mathrm{m}^{3}$. Calculations suggest the contribution from matrix yielding is modest, so a more accurate estimate of $W_{p}$ is not needed.

Fiber-matrix debonding

The energy from fiber-matrix debonding is the energy needed to break the fiber-matrix bond times the surface area of each debonded fiber. The fracture energy of the fiber-matrix bond has been estimated under mode II loading to be $\mathrm{b}^{34}$

$$
G_{I I c, i}=\frac{\sigma_{f d}^{2} d_{f}}{8 E_{f}}
$$

where $\sigma_{f d}$ is the stress in the fiber at the onset of debonding and $E_{f}$ is the fiber modulus (70 GPa). All fibers within $100 \mu \mathrm{m}$ of the fracture plane are assumed to debond completely, regardless of fiber orientation or whether the fiber intersects the fracture plane. The number of fibers within the debonded region per unit area has been estimated from

$$
N_{f d}=\frac{h_{d} V_{f}}{\pi r_{f}^{2} \bar{l}_{f}}
$$

where $h_{d}$ is the distance away from the fracture plane over which debonding occurs $(100 \mu \mathrm{m})$. The fiber surface area has been estimated from $2 \pi r_{f}\left(\bar{l}_{f}+r_{f}\right)$. Thus, the contribution from debonding is

$$
G_{D}=\frac{4 G_{I I c, i} h_{d} V_{f}}{r_{f} \bar{l}_{f}}\left(r_{f}+\bar{l}_{f}\right)
$$

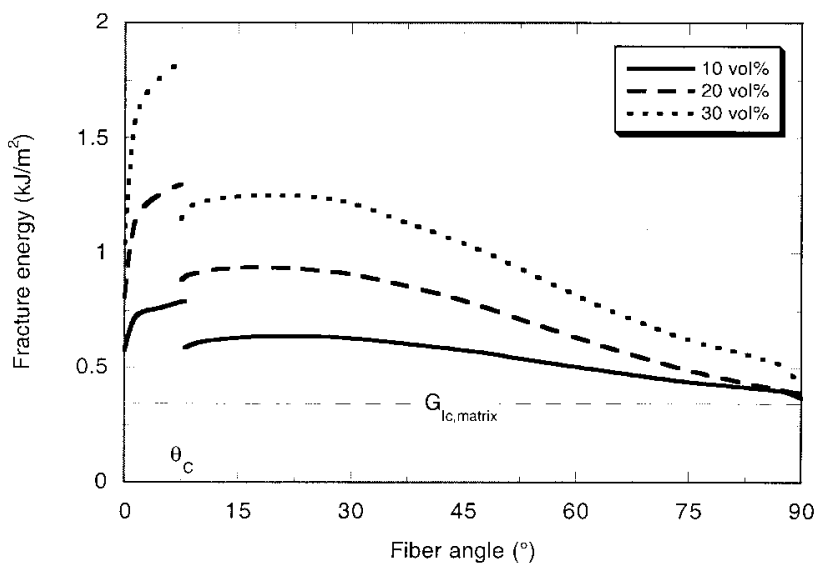

Figure 13 The predicted fracture energy vs. the polar fiber angle.

The mode II fracture energy of the fiber-matrix bond has been calculated from eq. (21), estimating $\sigma_{f d}$ from observations of fiber debonding within specimens loaded in flexure. Debonding was observed to occur at a composite stress of ca. $100 \mathrm{MPa}$, which corresponds to a fiber stress of ca. $600 \mathrm{MPa}$ using a shear lag model. This gives an interfacial fracture energy that is slightly less than $10 \mathrm{~J} / \mathrm{m}^{2}$, which is similar to the fracture energy reported by Andrews et al. for an epoxy-glass interface in the absence of moisture. ${ }^{35}$

Summing the contributions

The composite fracture energy is the sum of the various contributions. In addition to the toughening arising from the fibers, a term accounting for the fracture energy of the matrix has been included. This term is proportional to the volume fraction of the matrix. Combining this with eqs. (15)-(17), (20), and (23) gives for the composite fracture energy

$$
G_{I c, c}=\left(1-V_{f}\right) G_{I c, m}+G_{P O}+G_{B}+G_{Y}+G_{D}
$$

where the subscripts $c$ and $m$ refer to composite and matrix, respectively, and $G_{P O}$ is the sum of $G_{P O, a}$ and $G_{P O, b}$. The fracture energy of the matrix was $340 \mathrm{~J} / \mathrm{m}^{2}$. To calculate $G_{P O}$ [eqs. (15) and (16)], $\mu$ was assumed to be $1, r_{f}$ was $8 \mu \mathrm{m}$, values of $\sigma_{\curlyvee}$ were taken from Figure 10 , and $\sigma_{r}$ was estimated to be $27 \mathrm{MPa} .^{33}$ To calculate $G_{B}, U_{f u}$ was estimated to be $30 \mathrm{MJ} / \mathrm{m}^{3}$, and the number average fiber length was used for $\bar{l}_{f}, 70 \mu \mathrm{m}$. For calculating $G_{Y}, W_{p}$ was estimated to be $65 \mathrm{MJ} / \mathrm{m}^{3}$. To calculate $G_{D}, G_{I I c, i}$ was estimated to be $10 \mathrm{~J} / \mathrm{m}^{2}$ and $h_{d}$ was assumed to be $100 \mu \mathrm{m}$. Calculations of the composite fracture energy were numerically integrated, using the measured fiber orientation and length distributions.

The composite fracture energy is presented as a function of $\theta$ in Figure 13 for the fiber concentrations investigated. For this plot, the fracture energies were 
calculated assuming all of the fibers to be aligned at each angle, $\theta$. For $\theta \leq \theta_{c}$, the fracture energy increases with $\theta$ because of snubbing, and the fibers pull-out completely. At $\theta_{c}$ a discontinuity exists because above $\theta_{c}$ the fibers are predicted to break, and only the broken ends of the fibers pull-out. At higher angles, the fracture energy decreases with increasing $\theta$ because toughening from fiber pull-out and fiber breakage decrease as $\theta$ increases. The fracture energy approaches that of the matrix as $\theta$ approaches $90^{\circ}$, where fiber-matrix debonding is the only available toughening mechanism. The fracture energy increases with fiber concentration at all fiber angles below $90^{\circ}$ and is greatest at low fiber angles, especially near and below $\theta_{c}$.

Figure 13 may also be used to address the relative contributions of the different mechanisms over the complete range of $\theta$. The following is based on $20 \mathrm{vol}$ $\%$ fibers. For $\theta \leq \theta_{c}$, the primary toughening mechanisms are fiber pull-out and snubbing. The predicted contribution from pull-out at $\theta=0^{\circ}$ is $420 \mathrm{~J} / \mathrm{m}^{2}$, and the contribution from pull-out plus snubbing increases to $890 \mathrm{~J} / \mathrm{m}^{2}$ at $\theta_{c}$. For $\theta>\theta_{c}$, toughening from pull-out and snubbing decreases sharply as fibers break, and only the broken ends of the fibers pull-out. However, pull-out remains the most effective toughening mechanism just above $\theta_{c}$, contributing around $370 \mathrm{~J} / \mathrm{m}^{2}$ at $\theta=10^{\circ}$. Fiber breakage contributes roughly $140 \mathrm{~J} / \mathrm{m}^{2}$ at $\theta=10^{\circ}$. Contributions from fiber pull-out and fiber breakage decrease with increasing fiber angle and approach zero as $\theta=90^{\circ}$; this accounts for the decrease in predicted fracture energy with increasing $\theta$. Fiber-matrix debonding is predicted to contribute a modest $110 \mathrm{~J} / \mathrm{m}^{2}$ to the composite fracture energy, regardless of fiber angle. Matrix yielding adjacent to fibers bridging the crack gives the least

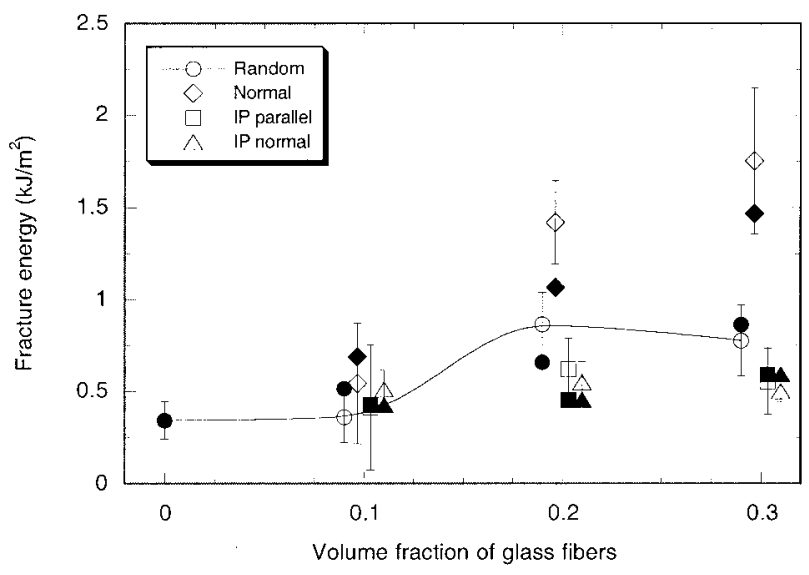

Figure 14 Comparison of measured and calculated fracture energy for all fiber concentrations and orientations. Open symbols are for measured values; filled symbols are for calculated values. Data at a common fiber concentration have been shifted horizontally to distinguish between fiber orientations. The line connecting the random data is included only as a guide.

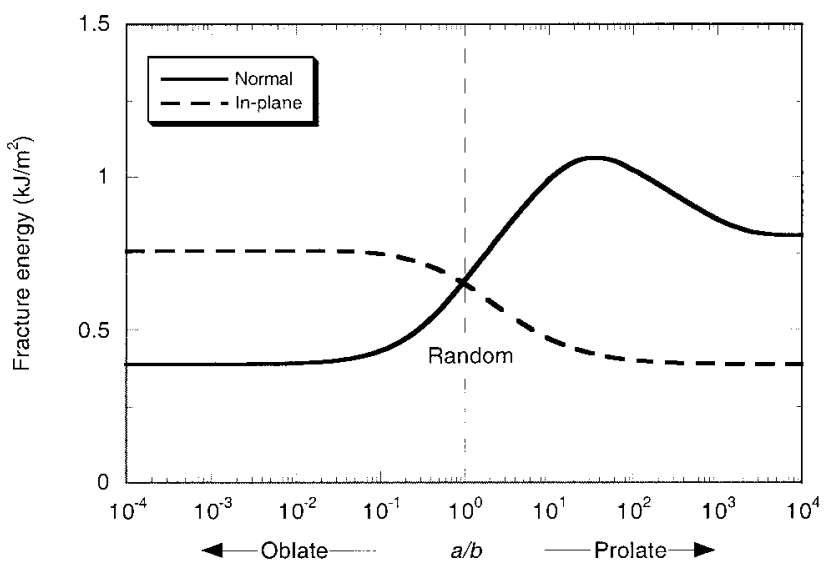

Figure 15 The predicted fracture energy versus the eccentricity of the ellipsoidal fiber angle distribution for $20 \mathrm{vol} \%$ fibers.

toughening, accounting for a maximum of ca. $80 \mathrm{~J} / \mathrm{m}^{2}$ at $\theta=30^{\circ}$. Fracture of the matrix at this fiber concentration contributes roughly another $250 \mathrm{~J} / \mathrm{m}^{2}$.

Calculated values of the fracture energy are compared with measured values in Figure 14. Open symbols indicate measured values, and filled symbols indicate calculated values. In general, the estimations from eq. (24) agree with the measured fracture energies. The normal-aligned specimens are estimated to give the greatest toughening. The nonaligned specimens are estimated to give less toughening than the normal-aligned. The two in-plane alignments are estimated to give the least toughening.

\section{Other fiber angle distributions}

The above calculations can be applied to other fiber angle distributions. These will be assumed to be ellipsoidal, with the major axis of the ellipsoid, $a$, being either normal to or within the fracture plane. The density of fibers oriented at any polar angle $\theta$ is proportional to the distance from the center to the surface of the ellipsoid at that angle. The predicted fracture energy vs. the eccentricity of the ellipsoidal distribution, which is defined as the ratio of the axes of the ellipsoid $(a / b)$, is shown in Figure 15 for a fiber concentration of $20 \mathrm{vol} \%$. The solid line is for the $a$-axis normal to the fracture plane, and the dashed line is for the $a$-axis within the fracture plane. For $a / b>1$, the fiber angle distribution is a prolate ellipsoid, and the fibers are predominantly aligned along the $a$-axis. For $a / b<1$, the distribution is oblate, and fibers tend to be perpendicular to the $a$-axis, radiating away from this axis. For $a / b=1$, the distribution is spherical (i.e., fibers are randomly oriented). For reference, the measured fiber angle distribution shown in Figure 4 for aligned specimens corresponds to an $a / b$ ratio of ca. 20 . For normal prolate distributions, the fracture energy increases with increasing $a / b$, reaching a maxi- 
mum ca. $1080 \mathrm{~J} / \mathrm{m}^{2}$ at $a / b$ ca. 40 ; the fracture energy then drops to ca. $810 \mathrm{~J} / \mathrm{m}^{2}$ as $a / b$ continues to increase.

This occurs because snubbing leads to greater toughening for fibers angled around $\theta_{c}$ than for those near $0^{\circ}$ (Fig. 13). For in-plane prolate distributions, the fracture energy decreases with increasing $a / b$, approaching $390 \mathrm{~J} / \mathrm{m}^{2}$ at very large $a / b$ ratios. This arises because the fibers become oriented within the fracture plane. For normal oblate distributions, the fracture energy is predicted to decrease with decreasing $a / b$, approaching $390 \mathrm{~J} / \mathrm{m}^{2}$ at very small $a / b$ ratios. As with the in-plane prolate distribution, this occurs because the fibers become aligned within the fracture plane. For in-plane oblate distributions, the fracture energy increases with decreasing $a / b$, approaching $760 \mathrm{~J} / \mathrm{m}^{2}$ at very small $a / b$ ratios. The fracture energy is lower than that of normal prolate distributions because not all fibers are aligned perpendicular to the fracture plane (fibers radiate from the $a$-axis). For $a / b=1$, the fracture energy is predicted to be ca. $660 \mathrm{~J} / \mathrm{m}^{2}$.

\section{CONCLUSIONS}

Toughening of polymers by either randomly oriented or aligned fibers that are shorter than their critical length has been used to investigate toughening mechanisms in short fiber-reinforced polymers. Fiber pullout appears to be the most effective toughening mechanism, and occurs completely for fibers nearly perpendicular to the fracture plane. Furthermore, snubbing is predicted to provide a significant contribution to pullout toughening. Fiber breakage is less effective than fiber pull-out and occurs for fibers with $\theta>\theta_{c}$ embedded more than about a fiber diameter. Toughening from fiber pull-out and fiber breakage decreases as the polar fiber angle increases, and the fracture energy of the composite approaches that of the neat polymer at $\theta=90^{\circ}$. Fiber-matrix debonding and localized matrix yielding adjacent to fibers that bridge the crack are estimated to give only modest toughening. Estimations of short fiber toughening based on the above mechanisms taking the measured fiber angle and length distributions into account corresponded with the fracture energies measured for the random and aligned composites. Predictions of toughening for a range of ellipsoidal fiber angle distributions suggest that snubbing can give rise to greater toughening for slightly misaligned fiber distributions than perfectly aligned distributions, when the alignment direction is perpendicular to the fracture plane.

\section{References}

1. De, S. K.; White, J. R., Eds. Short Fibre-Polymer Composites; Woodhead Publishing: Cambridge, 1996.

2. Jones, R. F. Guide to Short Fiber Reinforced Plastics; Hanser Publishers: Munich, 1998

3. Folkes, M. J. Short Fibre Reinforced Thermoplastics; Research Studies Press: New York, 1982.

4. Karger-Kocsis, J. In Application of Fracture Mechanics to Composite Materials; Friedrich, K., Ed.; Elsevier Science Publishers: Amsterdam, 1989, p. 189.

5. Friedrich, K.; Carlsson, L. A.; Gillespie, J. W.; Karger-Kocsis, J. In Thermoplastic Composite Materials; Carlsson, L. A., Ed.; Elsevier Science Publishers: Amsterdam, 1991, p. 233.

6. Mandell, J. F.; Darwish, A. Y.; McGarry, F. J. In Test Methods and Design Allowables for Fibrous Composites; Chamis, C. C., Ed.; American Society for Testing and Materials: Philadelphia, 1981, p. 73.

7. Friedrich, K. Compos Sci Technol 1985, 22, 43.

8. Hashemi, S.; Mugan, J. J Mater Sci 1993, 28, 3983.

9. Huang, D. D. Polym Compos 1995, 16, 10.

10. Lumini, F.; Pavan, A. Plast Rubber Compos Process Appl 1998, 27, 240.

11. Nilsson, G.; Ericson, M. L.; Holmberg, J. A. Polym Compos 2000, 21, 1007.

12. Benevolenski, O. I.; Karger-Kocsis, J. Compos Sci Technol 2001, 61, 2413.

13. Owen, M. J.; Whybrew, K. Plast Rubber 1976, 1, 231.

14. Hine, P. J.; Tsui, S. W.; Coates, P. D.; Ward, I. M.; Duckett, R. A. Composites A 1997, 28, 949.

15. Peng, J.; Lin, T. L.; Calvert, P. Composites A 1999, 30, 133.

16. McNally, D. Polym Plast Technol Eng 1977, 8, 101.

17. Nikpur, K.; Chen, Y. F.; Kardos, J. L. Compos Sci Technol 1990, $38,175$.

18. Helfet, J. L.; Harris, B. J Mater Sci 1972, 7, 494.

19. Park, C.; Robertson, R. E. Mater Sci Eng A 1998, 257, 295.

20. NIH Image program available on the internet at http://rsb. info.nih.gov/nih-image/.

21. Broek, D. Elementary Engineering Fracture Mechanics; Kluwer Academic Publishers: Dordrecht, 1986.

22. Tada, H. Stress Analysis of Cracks Handbook; Paris Productions; St. Louis, MO, 1985.

23. Hull, D.; Clyne, T. W. An Introduction to Composite Materials; University Press: Cambridge, 1996.

24. Voss, H.; Walter, R. J Mater Sci Lett 1985, 4, 1174.

25. Lauke, B.; Schultrich, B.; Barthel, R. Compos Sci Technol 1985, 23, 21.

26. Wells, J. K.; Beaumont, P. W. R. J Mater Sci 1988, 23, 1274.

27. Fu, S. Y.; Lauke, B. J Mater Sci 1997, 32, 1985.

28. Owen, M. J.; Bishop, P. T. J Compos Mater 1973, 7, 146.

29. Alexander, R. M.; Schapery, R. A.; Jerina, K. L.; Sanders, B. A. In Short Fiber Reinforced Composite Materials; Sanders, B. A., Ed.; American Society for Testing and Materials; Philadelphia, 1982, p. 208.

30. Mower, T. M.; Li, V. C. Eng Frac Mech 1987, 26, 593.

31. Li, V. C.; Wang, Y.; Backer, S. Composites 1990, 21, 132.

32. Morton, J.; Groves, G. W. J Mater Sci 1974, 9, 1436.

33. Harris, B. J Mater Sci 1978, 13, 173.

34. Outwater, J. O.; Murphy, M. C. Mod Plast 1970, 9, 160.

35. Andrews, E. H.; Pingsheng, H.; Vlachos, C. Proc R Soc Lond A 1982, 381, 345. 